CFNUL/01-04

DFAQ-2001/05-TH

\title{
Leptogenesis via Collisions: Leaking Lepton Number to the Hidden Sector
}

\author{
Luís Bento ${ }^{a}$ and Zurab Berezhiani ${ }^{b, c}$ \\ ${ }^{a}$ Centro de Física Nuclear da Universidade de Lisboa, \\ Avenida Prof. Gama Pinto 2, 1649-003 Lisboa, Portugal \\ ${ }^{b}$ Dipartamento di Fisica, Universitá di L'Aquila, I-67010 Coppito, AQ, Italy \\ INFN, Laboratori Nazionali del Gran Sasso, I-67010 Assergi, AQ, Italy \\ ${ }^{c}$ Andronikashvili Institute of Physics, GE-380077 Tbilisi, Georgia
}

(November, 2001)

\begin{abstract}
We propose a leptobaryogenesis mechanism in which the non-zero $B-L$ of the Universe is produced in out-of-equilibrium, lepton number and $\mathrm{CP}$ violating scattering processes that convert ordinary particles into particles of some hidden sector. In particular, we consider the processes $l \phi \rightarrow l^{\prime} \phi^{\prime}, \bar{l}^{\prime} \bar{\phi}^{\prime}$ mediated by the heavy Majorana neutrinos $N$ of the seesaw mechanism, where $l$ and $\phi$ are ordinary lepton and Higgs doublets and $l^{\prime}, \phi^{\prime}$ their hidden counterparts. Such a leptogenesis mechanism is effective even if the reheat temperature is much smaller than the heavy neutrino masses. In particular, it can be as low as $10^{9} \mathrm{GeV}$.
\end{abstract}

PACS numbers: 98.80.Cq., 11.30.Er., 11.30.Fs., 14.60.St

It is well known that a non-zero baryon asymmetry (BA) can be produced in the initially baryon symmetric universe if three conditions are fulfilled: B-violation, CP-violation and departure from thermal equilibrium [1]. These conditions can be satisfied in the decays of heavy particles of grand unified theories. On the other hand, the sphaleron processes, which violate $B+L$ but conserve $B-L$, are effective at temperatures from about $10^{12} \mathrm{GeV}$ down to $100 \mathrm{GeV} \mathrm{[2].} \mathrm{Thus,} \mathrm{one} \mathrm{actually}$ needs to produce a non-zero $B-L$ rather than just $B$, a fact that disfavors the simplest baryogenesis picture based on grand unification models like $S U(5)$. When sphalerons are in equilibrium, the baryon number and $B-L$ are related as $B=a(B-L)$, where $a$ is a model dependent order one coefficient 3]. Hence, in order to obtain the observed baryon to entropy density ratio $B=n_{B} / s=(0.6-1) \times 10^{-10}$, the produced $B-L$ needs to be $\mathcal{O}\left(10^{-10}\right)$.

The seesaw mechanism for neutrino masses offers an elegant possibility of generating non-zero $B-L$ in $\mathrm{CP}$ violating decays of heavy Majorana neutrinos $N$ into leptons and Higgses, the so called leptogenesis scenario [4]. Namely, due to complex Yukawa constants, the decay rates $\Gamma(N \rightarrow l \phi)$ and $\Gamma(N \rightarrow \bar{l} \bar{\phi})$ can be different from each other, so that leptons $l$ and anti-leptons $\bar{l}$ are produced in different amounts.

In this Letter we propose an alternative mechanism of leptogenesis that is based on scattering processes rather than particle decays. The main idea consists in the following. There may exist some hidden (shadow or mirror) sector of new particles which are not in thermal equilibrium with the ordinary particle world as far as the two systems interact very weakly e.g., if they only communicate via gravity. However, other messengers may well exist namely, superheavy gauge singlets like right-handed neutrinos which can mediate very weak effective interactions between the ordinary and shadow leptons. Then, a net $B-L$ could emerge in the Universe as a result of CPviolating effects in the unbalanced production of shadow particles from ordinary particle collisions.
The simplest model of this type can be described as follows. Consider the standard $S U(3) \times S U(2) \times U(1)$ model, containing three generations of leptons $l_{i}=(\nu, e)_{i}, e_{i}^{c}$ and quarks, the Higgs doublet $\phi$, and some heavy singlet fermions $N_{a}$. Imagine now, that there is also a hidden sector with gauge symmetry $G^{\prime}$, containing fermion and scalar fields that are singlets under the standard model gauge group, while the ordinary particles are instead singlets under $G^{\prime}$. The interesting candidate can be a mirror sector, exact duplicate of the observable sector with the same gauge symmetry $G^{\prime}=S U(3)^{\prime} \times S U(2)^{\prime} \times U(1)^{\prime}$ and with the same particle content [5]9]. However, in the more general case $G^{\prime}$ could be any gauge symmetry group containing, among other possible particles, fermions $l_{k}^{\prime}$ and scalar $\phi^{\prime}$ possessing opposite gauge charges so that the products $l_{k}^{\prime} \phi^{\prime}$ are gauge invariant.

In this case, the heavy singlet neutrinos $N$ can couple to $l, \phi$ as well as to $l^{\prime}, \phi^{\prime}$ and hence play the role of messengers between ordinary and shadow particles. The relevant Yukawa couplings have the form:

$$
h_{i a} l_{i} N_{a} \phi+h_{k a}^{\prime} l_{k}^{\prime} N_{a} \phi^{\prime}+\frac{1}{2} M_{a b} N_{a} N_{b}+\text { H.C. }
$$

(charge-conjugation matrix $C$ is omitted); the $l, N, l^{\prime}$ states are left handed while their $C$-conjugate, righthanded anti-particles are denoted as $\bar{l}, \bar{N}, \bar{l}^{\prime}$. Without loss of generality, the heavy neutrino mass matrix can be taken in diagonal basis as $M_{a}=g_{a} M, M$ being the overall mass scale and $g_{a}$ order one real constants. After integrating out the heavy neutrinos $N$ the effective operators emerge from the couplings (1) as

$$
\frac{A_{i j}}{2 M} l_{i} l_{j} \phi \phi+\frac{D_{i k}}{M} l_{i} l_{k}^{\prime} \phi \phi^{\prime}+\frac{A_{k n}^{\prime}}{2 M} l_{k}^{\prime} l_{n}^{\prime} \phi^{\prime} \phi^{\prime}+\text { H.C. , }
$$

with coupling constant matrices of the form $A=h g^{-1} h^{T}$, $A^{\prime}=h^{\prime} g^{-1} h^{\prime T}$ and $D=h g^{-1} h^{\prime T}$.

Our mechanism works within the following scenario. We assume that the initial densities of the ordinary and hidden sectors are different from each other. In particular, the reheat temperature of the hidden sector should 
be smaller than the visible one, $T_{R}^{\prime}<T_{R}$, which can be achieved in certain inflationary models $[10$. The two particle systems interact very weakly so that they do not come in thermal equilibrium with each other after reheating. The heavy neutrino masses are much larger than the reheat temperature $T_{R}$ and thus cannot be thermally produced. As a result, the usual leptogenesis mechanism via $N \rightarrow l \phi$ decays is ineffective 11. Now, the important role is played by lepton number violating scatterings mediated by the heavy neutrinos $N$ which stay out of equilibrium once $T_{R} \ll M$. On the other hand, they violate $\mathrm{CP}$ due to complex Yukawa couplings in Eq. (1).

In other words, we assume that after the postinflationary reheating, different temperatures are established in the two sectors: the hidden world is cooler or ultimately, completely "empty". Nevertheless, it starts to be "slowly" occupied due to the leaking of entropy from the ordinary sector through the reactions $l_{i} \phi \rightarrow \bar{l}_{k}^{\prime} \bar{\phi}^{\prime}$, $\bar{l}_{i} \bar{\phi} \rightarrow l_{k}^{\prime} \phi^{\prime}$. Then, because of CP violation, the crosssections with leptons and anti-leptons in the initial state are different from each other. As a result, leptons leak to the hidden sector more (or less) effectively than antileptons and a non-zero $B-L$ is produced in the Universe.

A temperature scale that plays a crucial role in our considerations is the reheat temperature $T_{R}$, at which the inflaton decay and entropy production of the Universe is over and under which the Universe is dominated by a relativistic plasma of ordinary particle species.

It is convenient to introduce a parameter that characterizes the reaction rate per Hubble time at the temperature $T=T_{R}, K=(\Gamma / 2 H)_{R}$. Here $H=1.66 g_{*}^{1 / 2} T^{2} / M_{P l}$ is the Hubble parameter and $g_{*}$ is the effective number of particle degrees of freedom. For the $\Delta L=1$ reaction rate we have $\Gamma_{1}=\sigma_{1} n_{e q}$, where $n_{e q} \simeq\left(1.2 / \pi^{2}\right) T^{3}$ is an equilibrium density per degree of freedom and $\sigma_{1}$ is the total cross section of $l \phi \rightarrow \bar{l}^{\prime} \bar{\phi}^{\prime}$ scatterings,

$$
\sigma_{1}=\sum \sigma\left(l \phi \rightarrow \bar{l}^{\prime} \bar{\phi}^{\prime}\right)=\frac{Q_{1}}{8 \pi M^{2}} .
$$

The sum is taken over all flavor and isospin indices of initial and final states, and $Q_{1}=\operatorname{Tr}\left(D^{\dagger} D\right)=$ $\operatorname{Tr}\left[\left(h^{\prime \dagger} h^{\prime}\right) g^{-1}\left(h^{\dagger} h\right)^{*} g^{-1}\right]$. Hence, the out-of-equilibrium condition for this process reads as

$$
K_{1}=\left(\frac{\Gamma_{1}}{2 H}\right)_{R} \simeq 1.5 \times 10^{-3} \frac{Q_{1} T_{R} M_{P l}}{g_{*}^{1 / 2} M^{2}}<1,
$$

which, for a given reheat temperature $T_{R}$, translates into the lower limit on the heavy neutrino mass scale $M$ :

$$
M_{12}>1.3 Q_{1}^{1 / 2} T_{9}^{1 / 2}
$$

where $M_{12} \equiv\left(M / 10^{12} \mathrm{GeV}\right), T_{9} \equiv\left(T_{R} / 10^{9} \mathrm{GeV}\right)$ and we have taken $g_{*} \approx 100$ as in the standard model.

However, there are also scattering processes like $l \phi \rightarrow$ $\bar{l} \bar{\phi}$ etc., which can wash out the produced $B-L$ unless they are out of equilibrium [1, 12]. The total rate of $\Delta L=2$ processes is given by $\Gamma_{2} \simeq\left(3 Q_{2} / 4 \pi M^{2}\right) n_{\mathrm{eq}}$ where $Q_{2}=\operatorname{Tr}\left(A^{\dagger} A\right)=\operatorname{Tr}\left[\left(h^{\dagger} h\right) g^{-1}\left(h^{\dagger} h\right)^{*} g^{-1}\right]$. Therefore, the condition $K_{2}=\left(\Gamma_{2} / 2 H\right)_{R}<1$ translates into a
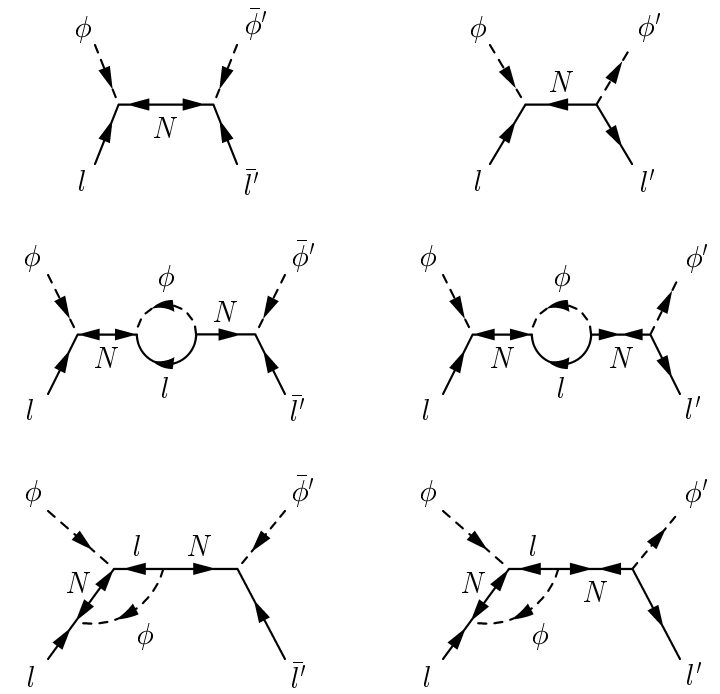

FIG. 1. Tree-level and one-loop diagrams contributing to the CP-asymmetries in $l \phi \rightarrow \bar{l}^{\prime} \bar{\phi}^{\prime}$ (left column) and $l \phi \rightarrow l^{\prime} \phi^{\prime}$ (right column).

lower bound similar to (5),

$$
M_{12}>3.2 Q_{2}^{1 / 2} T_{9}^{1 / 2} .
$$

Clearly, if the Yukawa constants $h_{i a}$ and $h_{k a}^{\prime}$ are of the same order, the out-of-equilibrium conditions for $\Delta L=1$ and $\Delta L=2$ processes are nearly equivalent to each other.

Let us turn now to $\mathrm{CP}$ violation. In $\Delta L=1$ processes the CP-odd lepton number asymmetry emerges from the interference between the tree-level and one-loop diagrams of Fig. 1. The tree-level amplitude for the dominant channel $l \phi \rightarrow \bar{l}^{\prime} \bar{\phi}^{\prime}$ goes as $1 / M$ and the radiative corrections as $1 / M^{3}$. For the channel $l \phi \rightarrow l^{\prime} \phi^{\prime}$ instead, both tree-level and one-loop amplitudes go as $1 / M^{2}$. As a result, the cross section CP-asymmetries are the same for both $l \phi \rightarrow \bar{l}^{\prime} \bar{\phi}^{\prime}$ and $l \phi \rightarrow l^{\prime} \phi^{\prime}$ channels (on the contrary, the diagrams with $l^{\prime} \phi^{\prime}$ inside the loops, not shown in Fig. 1. yield asymmetries, $\pm \Delta \sigma^{\prime}$, symmetric to each other). However, $\mathrm{CP}$ violation takes also place in $\Delta L=2$ processes (see Fig. 2). This is a consequence of the very existence of the hidden sector namely, the contribution of the hidden particles to the one-loop diagrams of Fig. 2. The direct calculation gives:

$$
\begin{aligned}
& \sigma\left(l \phi \rightarrow \bar{l}^{\prime} \bar{\phi}^{\prime}\right)-\sigma\left(\bar{l} \bar{\phi} \rightarrow l^{\prime} \phi^{\prime}\right)=\left(-\Delta \sigma-\Delta \sigma^{\prime}\right) / 2, \\
& \sigma\left(l \phi \rightarrow l^{\prime} \phi^{\prime}\right)-\sigma\left(\bar{l} \bar{\phi} \rightarrow \bar{l}^{\prime} \bar{\phi}^{\prime}\right)=\left(-\Delta \sigma+\Delta \sigma^{\prime}\right) / 2, \\
& \sigma(l \phi \rightarrow \bar{l} \bar{\phi})-\sigma(\bar{l} \bar{\phi} \rightarrow l \phi)=\Delta \sigma \\
& \Delta \sigma=\frac{3 J S}{32 \pi^{2} M^{4}},
\end{aligned}
$$

where $J=\operatorname{Im} \operatorname{Tr}\left[\left(h^{\prime \dagger} h^{\prime}\right) g^{-2}\left(h^{\dagger} h\right) g^{-1}\left(h^{\dagger} h\right)^{*} g^{-1}\right]$ is the $\mathrm{CP}$-violation parameter and $S$ is the c.m. energy square ( $\Delta \sigma^{\prime}$ is obtained from $\Delta \sigma$ by exchanging $h$ with $h^{\prime}$ ).

This is in perfect agreement with CPT invariance that requires that the total cross sections for particle and antiparticle scatterings are equal to each other: $\sigma(l \phi \rightarrow X)=$ $\sigma(\bar{l} \bar{\phi} \rightarrow X)$. Indeed, taking also into account that by 

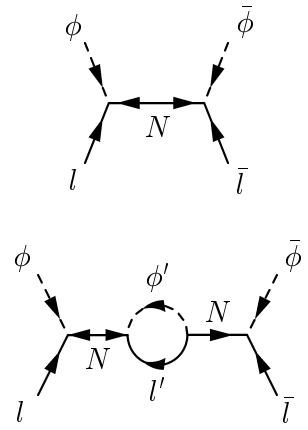
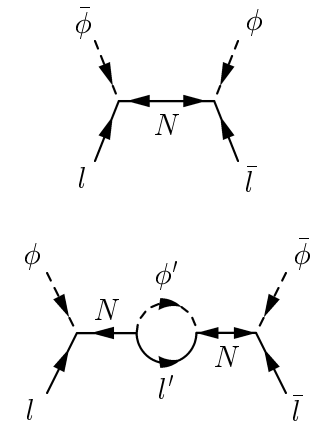

FIG. 2. Tree-level and one-loop diagrams contributing to the CP-asymmetry of $l \phi \rightarrow \bar{l} \bar{\phi}$. The external leg labels identify the initial and final state particles.

$\mathrm{CPT}, \sigma(l \phi \rightarrow l \phi)=\sigma(\bar{l} \bar{\phi} \rightarrow \bar{l} \bar{\phi})$, we obtain that the $\mathrm{CP}$ asymmetries in the $\Delta L=1$ and $\Delta L=2$ processes should be related as follows:

$$
\begin{aligned}
& \sigma\left(l \phi \rightarrow X^{\prime}\right)-\sigma\left(\bar{l} \bar{\phi} \rightarrow X^{\prime}\right)+ \\
& \sigma(l \phi \rightarrow \bar{l} \bar{\phi})-\sigma(\bar{l} \bar{\phi} \rightarrow l \phi)=0,
\end{aligned}
$$

where $X^{\prime}$ are the hidden sector final states, $\bar{l}^{\prime} \bar{\phi}^{\prime}$ and $l^{\prime} \phi^{\prime}$. That is, the $\Delta L=1$ and $\Delta L=2$ reactions have $\mathrm{CP}$ asymmetries with equal intensities but opposite signs. But, as $L$ varies in each case by a different amount, a net lepton number decrease is produced, or better, a net increase of $B-L \propto \Delta \sigma$.

Contrary to the lepton number that is violated by sphaleron processes, $B-L$ is only violated by the above kind of reactions. As long as we assume that the hidden sector is essentially depleted of particles, the only relevant reactions are the ones with ordinary particles in the initial state. Hence, the evolution of the $B-L$ number density is determined by the $\mathrm{CP}$ asymmetries shown in Eqs. (7) and obeys the equation

$$
\frac{d n_{B-L}}{d t}+3 H n_{B-L}=\frac{3}{4} \Delta \sigma n_{\mathrm{eq}}^{2} .
$$

Since the CP-asymmetric cross section $\Delta \sigma$ is proportional to the thermal average c.m. energy square $S \simeq$ $17 T^{2}$ and $H=1 / 2 t \propto T^{2}$, one integrates the above equation from $T=T_{R}$ to the low temperature limit and obtains the final $B-L$ asymmetry of the Universe as

$$
B-L=\frac{n_{B-L}}{s}=\left[\frac{\Delta \sigma n_{\mathrm{eq}}^{2}}{4 H s}\right]_{R},
$$

where $s$ is the entropy density.

The following remark is in order. In fact, the lepton number production starts as soon as the inflaton starts to decay and the particle thermal bath is produced, before the reheat temperature is established. (Recall that the maximal temperature at the reheating period is usually larger than $T_{R}$.) In this epoch the Universe is still dominated by the inflaton oscillations and therefore it expands as $t^{2 / 3}$ while the entropy of the Universe grows as $t^{5 / 4}$. The integration of Eq. (9) from some higher temperatures down to $T_{R}$ gives an asymmetry 1.5 times larger than the estimation (10). Taking all these into account, the final result can be recasted as follows:

$$
B-L \approx 2 \times 10^{-3} \frac{J M_{P l} T_{R}^{3}}{g_{*}^{3 / 2} M^{4}} \approx 2 \times 10^{-8} \frac{J T_{9}^{3}}{M_{12}^{4}},
$$

where we have taken again $g_{*} \approx 100$. Taking also into account the lower limits (5) and (6), we obtain the upper limit on the produced $B-L$ :

$$
B-L<10^{-8} \frac{J T_{9}}{Q^{2}} ; \quad Q=\max \left\{Q_{1}, 6 Q_{2}\right\} .
$$

This shows that for Yukawa constants spread e.g. in the range $0.1-1$ one can achieve $B-L=\mathcal{O}\left(10^{-10}\right)$ for a reheat temperature as low as $T_{R} \sim 10^{9} \mathrm{GeV}$. Interestingly, this coincidence with the upper bound from the thermal gravitino production, $T_{R}<4 \times 10^{9} \mathrm{GeV}$ or so [13], indicates that our scenario could also work in the context of supersymmetric theories. Certainly, in a nonsupersymmetric theory $T_{R}$ can be much larger.

The hidden sector may include coupling constants (e.g. gauge coupling constants of $G^{\prime}$ ) large enough to thermalize the hidden particles at a temperature $T^{\prime}$. Once $K_{1}<1, T^{\prime}$ will be smaller than the parallel temperature of the ordinary system $T$. Obviously, the presence of the out-of-equilibrium hidden sector does not affect much the Big Bang Nucleosynthesis (BBN) epoch. Indeed, if the two sectors do not come into full thermal equilibrium at temperatures $T \sim T_{R}$ then, they evolve independently during the Universe expansion and approach the nucleosynthesis era with different temperatures. For $K_{1}<1$, the energy density transferred to the hidden sector will be crudely $\rho^{\prime} \approx\left(8 K_{1} / g_{*}\right) \rho$, where $g_{*}(\approx 100)$ is attained to the leptogenesis epoch. Thus, assuming that at the $\mathrm{BBN}$ epoch the shadow sector is dominated by relativistic degrees of freedom, we obtain an effective number of extra light neutrinos $\Delta N_{\nu} \approx K_{1} / 2$.

Observe, that our model can induce the masses of both the ordinary and shadow neutrinos via their seesaw mixing with the heavy Majorana neutrinos provided that the shadow Higgs $\phi^{\prime}$ has a vacuum expectation value (VEV) $v^{\prime} \ll M$. The first operator in Eq. (2), due to the ordinary Higgs $\operatorname{VEV}\left\langle\phi^{0}\right\rangle=v \sim 100 \mathrm{GeV}$, induces the small Majorana masses of the ordinary neutrinos while the other operators induce the mass and mixing mass terms of the shadow neutrinos contained in $l^{\prime}$ (in fact sterile neutrinos for the ordinary observer) with the ordinary active neutrinos [6,7. The total mass matrix of active-sterile neutrinos reads as [7]

$$
M_{\nu}=\left(\begin{array}{cc}
m_{\nu} & m_{\nu \nu^{\prime}} \\
m_{\nu \nu^{\prime}}^{T} & m_{\nu^{\prime}}
\end{array}\right)=\frac{1}{M}\left(\begin{array}{cc}
A v^{2} & D v v^{\prime} \\
D^{T} v v^{\prime} & A^{\prime} v^{\prime 2}
\end{array}\right) .
$$

In other words, it provides a simple explanation of why sterile neutrinos could be light (on the same grounds as the active neutrinos) and could have significant mixing with the ordinary neutrinos. For example, if $v^{\prime} \sim 10^{2} v$ then, the shadow neutrinos $\nu^{\prime}$ with masses of $\mathrm{keV}$ order could provide the warm dark matter component in the Universe [8]. Instead, if $\left\langle\phi^{\prime}\right\rangle=0$, the $\nu^{\prime}$ are massless and unmixed with the ordinary neutrinos. 
It is worth noticing that the same mechanism that produces the lepton number in the ordinary Universe, can also produce the lepton prime asymmetry in the hidden sector. The amount of this asymmetry will depend on the CP-violation parameter that replaces $J$ in Eqs. (7) and $\Delta \sigma^{\prime}$ namely, $J^{\prime}=\operatorname{Im} \operatorname{Tr}\left[\left(h^{\dagger} h\right) g^{-2}\left(h^{\prime \dagger} h^{\prime}\right) g^{-1}\left(h^{\prime \dagger} h^{\prime}\right)^{*} g^{-1}\right]$. Then, if the shadow sector contains also some heavier particles of the lepton or baryon type, the shadow matter could provide a dark matter.

The interesting candidate is the mirror world, which has attracted a significant interest over the last years, being motivated by various problems in particle physics and cosmology [5 9]. In this case we have a theory given by the product $G \times G^{\prime}$ of two identical gauge factors with identical particle contents, which could naturally emerge e.g. in the context of $E_{8} \times E_{8}^{\prime}$ superstring theories. In particular, the $G$ sector contains ordinary particles $\phi$, $l$, etc., whereas $G^{\prime}$ contains their mirror partners $\phi^{\prime}, l^{\prime}$, etc., in equivalent representations. It is natural to assume that both particle sectors are described by identical Lagrangians, that is, all coupling constants (gauge, Yukawa, Higgs) have the same pattern in both sectors and thus their microphysics is the same.

In particular, a discrete symmetry under the exchange $\phi \rightarrow \phi^{\prime \dagger}, l \rightarrow \bar{l}^{\prime}$, etc., the so-called mirror parity, implies $h_{i a}^{\prime}=h_{i a}^{*}$. In this case the CP-violation parameters are the same, $J^{\prime}=J$. Then, one expects that $n_{B-L}=n_{B-L}^{\prime}$ and the mirror baryon number density should be equal to the ordinary baryon density, $\Omega_{B}^{\prime}=\Omega_{B}$. The mirror parity could be also spontaneously broken by the difference in weak scales $\langle\phi\rangle=v$ and $\left\langle\phi^{\prime}\right\rangle=v^{\prime}$, which would lead to somewhat different particle physics in the mirror sector [7,8, , e.g. the mirror leptons and baryons could be heavier (or lighter) than the ordinary ones. But, as the mechanism only depends on the Yukawa constant pattern in (1), one still has $n_{B-L}=n_{B-L}^{\prime}$, while $\Omega_{B}^{\prime} \neq \Omega_{B}$. Generically, the mirror sector provides a sort of self-interacting dark matter, however, if it is significantly colder than the visible one, $T^{\prime} / T<0.3$ or so, the mirror photons decouple early and the mirror matter would behave as a cold dark matter as far as the large scale formation is concerned [9]. Moreover, the mirror group $S U(2)^{\prime} \times U(1)^{\prime}$ may be even fully broken by a set of two or more Higgs VEVs, which would make the mirror photon a massive particle and thus the corresponding interaction short-range.

It is worthwhile to stress that the leptogenesis mechanism we propose does not really rely on model dependent features of the hidden sector. They are however important and in principle testable to some extent if the hidden sector is to make up for the dark matter of the Universe. Depending on the gauge structure, field content and symmetry breaking scales in the hidden sector, one could have a shadow matter behaving as a cold, warm or self-interacting dark matter, or their combination. The possible marriage between dark matter and the leptobaryogenesis mechanism is certainly an atractive feature of our scheme which deserves to be explored in more detail.

Let us conclude with the following remark. The magnitude of the produced $B-L$, Eq. (11), strongly depends on the temperature - namely, larger $B-L$ will be produced in the patches where the plasma is hotter. In the cosmological context, this would lead to a situation where apart from the adiabatic density/temperature perturbations, there also emerge correlated isocurvature fluctuations with variable $B$ and $L$ which could be tested with the future data on the CMB anisotropies and large scale structure.

We thank A. Dolgov and E. Paschos for discussions. We acknowledge Fundação para a Ciência e Tecnologia (FCT) for grant CERN/P/FIS/40129/2000. The work of Z. B. was partially supported by the MURST research grant "Astroparticle Physics".

[1] A. D. Sakharov, JETP Lett. 5, 24 (1967).

[2] V. A. Kuzmin, V. A. Rubakov, M. E. Shaposhnikov, Phys. Lett. 155B, 36 (1985).

[3] For a review, see A. D. Dolgov, Phys. Rep. 222, 309 (1992); A. Riotto and M. Trodden, Annu. Rev. Nucl. Part. Sci. 49, 35 (1999); W. Buchmüller and M. Plümacher, Int. J. Mod. Phys. A 15, 5047 (2000).

[4] M. Fukugita, T. Yanagida, Phys. Lett. B 174, 45 (1986); Phys. Rev. D 42, 1285 (1990).

[5] T. D. Li and C. N. Yang, Phys. Rev. 104, 254 (1956); I. Yu. Kobzarev, L. B. Okun, I. Ya. Pomeranchuk, Sov. J. Nucl. Phys. 3, 837 (1966) [Yad. Fiz. 3, 1154 (1966)]; M. Pavšič, Int. J. Theor. Phys. 9, 229 (1974); S. I. Blinnikov and M. Yu. Khlopov, Sov. Astron. 27, 371 (1983) [Astron. Zh. 60, 632 (1983)]; M. Yu. Khlopov et al., Sov. Astron. 35, 21 (1991) [Astron. Zh. 68, 42 (1991)]; R. Foot, H. Lew and R. R. Volkas, Phys. Lett. B 272, 67 (1991); Mod. Phys. Lett. A 7, 2567 (1992); H. M. Hodges, Phys. Rev. D 47, 456 (1993); Z. K. Silagadze, Phys. At. Nucl. 60, 272 (1997); Z. Berezhiani, Phys. Lett. B 417, 287 (1998).

[6] R. Foot and R. R. Volkas, Phys. Rev. D 52, 6595 (1995); For earlier work, R. Foot, H. Lew and R. R. Volkas, Mod. Phys. Lett. A 7, 2567 (1992).

[7] Z. G. Berezhiani and R. N. Mohapatra, Phys. Rev. D 52, 6607 (1995); For earlier work, E. Kh. Akhmedov, Z. G. Berezhiani and G. Senjanović, Phys. Rev. Lett. 69, 3013 (1992).

[8] Z. G. Berezhiani, A. D. Dolgov and R. N. Mohapatra, Phys. Lett. B 375, 26 (1996); Z. G. Berezhiani, Acta Phys. Pol. B 27, 1503 (1996); V. S. Berezinsky, A. Vilenkin, Phys. Rev. D 62, 083512 (2000).

[9] Z. Berezhiani, D. Comelli and F. L. Villante, Phys. Lett. B 503, 362 (2001).

[10] E. Kolb, D. Seckel and M. Turner, Nature 514, 415 (1985).

[11] The heavy neutrinos $N$ could be produced also nonthermally, due to non-perturbative decay of inflaton oscillations; see G. F. Giudice et al., JHEP 9908, 014 (1999). However, this possibility is model dependent and we do not consider it here.

[12] M. A. Luty, Phys. Rev. D 45, 455 (1992); M. Flanz and E. A. Paschos, Phys. Rev. D 58, 113009 (1998).

[13] J. R. Ellis, A. D. Linde and D. V. Nanopoulos, Phys. Lett. 118B, 59 (1982); D. V. Nanopoulos, K. A. Olive and M. Srednicki, ibid. 127B, 30 (1983). 